\title{
HH 1158: THE LOWEST LUMINOSITY EXTERNALLY IRRADIATED HERBIG-HARO JET
}

\author{
B. RIAZ ${ }^{1}$ AND E. T. WHELAN ${ }^{2,3}$ \\ ${ }^{1}$ Max-Planck-Institut für Extraterrestrische Physik, Giessenbachstrasse 1, D-85748 Garching, Germany \\ 2 Institute für Astronomie und Astrophysik, Eberhard Karls University Tuebingen, Sand 1, D-72076 Tübingen, Germany \\ Received 2015 October 18; accepted 2015 November 28; published 2015 December 18
}

\begin{abstract}
We have identified a new externally irradiated Herbig-Haro $(\mathrm{HH})$ jet, $\mathrm{HH} 1158$, within 2 pc of the massive OB type stars in the $\sigma$ Orionis cluster. At an $L_{\mathrm{bol}} \sim 0.1 L_{\odot}, \mathrm{HH} 1158$ is the lowest luminosity irradiated $\mathrm{HH}$ jet identified to date in any cluster. Results from the analysis of high-resolution optical spectra indicate asymmetries in the brightness, morphology, electron density, velocity, and the mass outflow rates for the blue and redshifted lobes. We constrain the position angle of the $\mathrm{HH} 1158$ jet at $102^{\circ} \pm 5^{\circ}$. The mass outflow rate and the mean accretion rate for $\mathrm{HH} 1158 \mathrm{using}$ multiple diagnostics are estimated to be $(5.2 \pm 2.6) \times 10^{-10} M_{\odot} \mathrm{yr}^{-1}$ and $(3.0 \pm 1.0)$ $\times 10^{-10} M_{\odot} \mathrm{yr}^{-1}$, respectively. The properties for $\mathrm{HH} 1158$ are notably similar to the externally irradiated $\mathrm{HH}$ 444-HH 447 jets previously identified in $\sigma$ Orionis. In particular, the morphology is such that the weaker jet beam is tilted toward the massive stars, indicating a higher extent of photo-evaporation. The high value for the $\mathrm{H} \alpha /[\mathrm{S}$ II] ratio is also consistent with the ratios measured in other irradiated jets, including $\mathrm{HH} \mathrm{444-HH} \mathrm{447.} \mathrm{The} \mathrm{presence} \mathrm{of}$ an extended collimated jet that is bipolar and the evidence of shocked emission knots make HH 1158 the first unique case of irradiated $\mathrm{HH}$ jets at the very low-luminosity end, and provides an opportunity to learn the physical properties of very faint $\mathrm{HH}$ jet sources.
\end{abstract}

Key words: Herbig-Haro objects - ISM: jets and outflows - stars: low-mass

\section{INTRODUCTION}

Herbig-Haro $(\mathrm{HH})$ objects are collisionally excited nebulae produced by highly collimated outflows ejected from young stellar objects (YSOs). Most HH jet sources are embedded within opaque cloud cores that obscure the immediate vicinity of the outflow sources. A case in exception are externally irradiated $\mathrm{HH}$ jets identified in $\mathrm{H}$ II regions or located near massive OB type stars. The external ultraviolet (UV) radiation field ionizes and photoablates the bulk of the gas surrounding the YSOs, and contributes to the excitation and visibility of the outflow (Reipurth et al. 1998; Reipurth \& Bally 2001).

Among the first such cases of externally irradiated $\mathrm{HH}$ jets were $\mathrm{HH} 444-\mathrm{HH} 447$, identified in the $\sigma$ Orionis ( $\sigma$ Ori) cluster (Reipurth et al. 1998). The $\sigma$ Ori cluster belongs to the Orion OB $1 \mathrm{~b}$ association. It is centered around the massive O9.5-type multiple star system of the same name, and has been suggested as an $\mathrm{H}$ II region (e.g., Walter et al. 2008). The $\mathrm{HH}$ $444-\mathrm{HH} 447$ jets are located within $\sim 2 \mathrm{pc}$ of $\sigma$ Ori (Figure 1), and are driven by optically visible $\mathrm{T}$ Tauri stars. Interestingly, all four jets are highly asymmetric, with the beam facing toward $\sigma$ Ori being much weaker and fainter than the counter beam, indicating a higher extent of photo-evaporation due to the ionizing radiation from the massive stars (Reipurth et al. 1998; Andrews et al. 2004). The sources of the irradiated jets are considered to be in an evolutionary state where they have retained tenuous or no envelope material, thereby exposing the disks to photo-erosion. The production of jets seems to have been unaffected. The main utility of such irradiated jets is that the radiation field can improve their visibility both close to and far from the shock region, thus enabling unambiguous determination of the jet physical parameters.

In this paper, we report the identification of a new irradiated jet, HH 1158, in the $\sigma$ Ori cluster. The driving source of $\mathrm{HH}$

\footnotetext{
${ }^{3}$ National University of Ireland, Maynooth, Ireland.
}

1158 is a very low-luminosity $\left(L_{\mathrm{bol}}=0.16 \pm 0.03 L_{\odot}\right)$ star, named Mayrit 1082188 (M1082188). In Riaz et al. (2015), we presented multi-wavelength optical through sub-millimeter observations for this YSO, including low-resolution optical spectroscopy. M1082188 is classified as a Flat Spectrum object. The Class Flat sources are considered to be at an intermediate stage between Class I and II and have tenuous envelopes compared to Class I objects (Greene et al. 1994). The proximity (within $\sim 2 \mathrm{pc}$ ) of M1082188 to the massive $\sigma$ Ori quintuple system (Figure 1) suggests that the ionizing radiation from nearby $\mathrm{OB}$ stars has stripped the circumstellar envelope surrounding the driving source of this irradiated jet, making it visible at optical and near-infrared wavelengths. The total (dust+gas) mass for this system as derived from the submillimeter fluxes is $\sim 22 M_{\text {Jup }}$. The optical spectrum exhibits prominent emission in the [O I] $\lambda \lambda 6300,6363 \AA$, [S II] $\lambda \lambda 6716$, $6730 \AA$, and the $\left[\mathrm{N}_{\mathrm{II}}\right] \lambda 6583 \AA$ forbidden emission lines (FELs), indicating that it is driving an outflow. Here, we present new results from an analysis of high-resolution spectra for HH 1158 obtained at the Very Large Telescope, using the UV-visual Echelle Spectrometer (UVES). Interestingly, HH 1158 shows a similar bipolar asymmetric jet morphology as observed for the HH 444-HH 447 jets, and is the lowest luminosity $\mathrm{HH}$ jet identified yet in the $\sigma$ Ori or any other cluster. While numerous example of jets from Class II very low-mass stars and brown dwarfs have now been studied (e.g., Whelan et al. 2009), these jets are considered to be analogous to the "micro-jets" driven by classical $\mathrm{T}$ Tauri stars, and no definite detection of $\mathrm{HH}$ objects associated with these jets have been made prior to this work. Thus this work provides us with a new way in which to study $\mathrm{HH}$ jet phenomena at the lowest masses. In Section 2, we give details of the new observations, the results from the spectral analysis are presented in Section 3, and the similarities between $\mathrm{HH} 1158$ and $\mathrm{HH}$ 444-HH 447 jets are discussed in Section 4. 


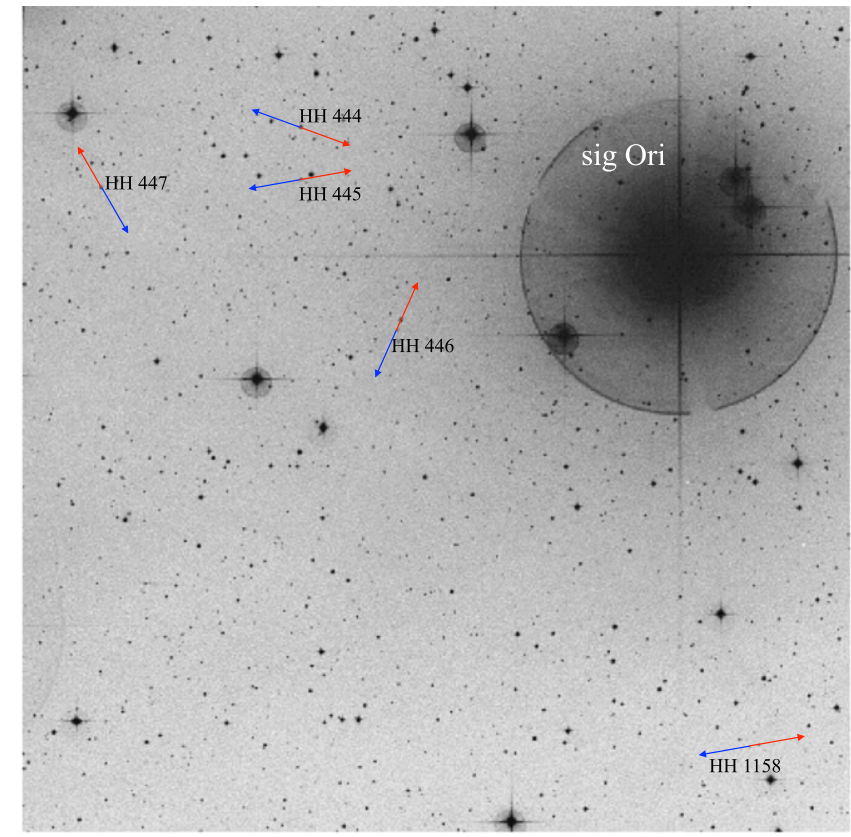

Figure 1. UKIDSS $K_{s}$-band image showing $\sigma$ Ori, the $\mathrm{HH} 444-\mathrm{HH} 447$, and $\mathrm{HH} 1158$ jets. The orientation of the blue and red lobes for the jets are marked as blue and red arrows, respectively. The scale of the image is about $30^{\prime} \times 30^{\prime}$. North is up, east is to the left.

\section{OBSERVATIONS AND DATA REDUCTION}

The UVES high-resolution spectra for HH 1158 (Program ID: 094.C-0667(A)) were obtained in 2014 September. We used the standard DIC2 $(437+760)$ setting, with cross dispersers of CD\#2 (HER_5) and CD\#4 (BK7_5). This setup provided a wavelength coverage from 373 to $946 \mathrm{~nm}$ in one exposure. The slit width was set to $1^{\prime \prime}$, resulting in a spectral resolution of $R \sim 40,000$. We obtained two spectra for the target, one per slit orientation at a position angle (P. A.) of $0^{\circ}$ and $90^{\circ}$. The total on-source exposure time was $3200 \mathrm{~s}$, split into two exposures. The seeing was recorded to be between 0 ". 5 and $1^{\prime \prime}$ during the nights when these observations were made. The UVES spectra were reduced using the ESO Reflex pipeline. The combined $0^{\circ}$ and $90^{\circ}$ spectra are shown in Figure 2; the fluxes measured in notable accretion and outflow diagnostics are listed in Table 1 . We estimate a signal-to-noise ratio $(\mathrm{S} / \mathrm{N})$ of better than $\sim 20$ in the red arm and $\sim 10$ in the blue part of the combined spectrum.

For the position-velocity (PV) diagrams and the spectroastrometric analysis, we have used the standard IRAF routines for continuum and sky line subtraction and for Gaussian fitting. Spectro-astrometry is a technique by which Gaussian fitting of the spatial profile of a spectrum as a function of wavelength is used to recover spatial information from spatially unresolved emission lines. It has been successfully used to disentangle outflow components to emission lines tracing accretion and to detect brown dwarf outflows (Whelan et al. 2005). We have followed the same process for the spectro-astrometric analysis as outlined in (Whelan \& Garcia 2008).

\section{RESULTS}

The optical spectrum for $\mathrm{HH} 1158$ exhibits all of the wellknown outflow-associated [O I], [S II], [N II], and [Fe II] FELs (Figure 2). There is also a marginal detection of the $[\mathrm{N}$ II] $\lambda$
$6548 \AA$ feature clearly resolved from the $\mathrm{H} \alpha$ line. This FEL is rarely seen in spectra of very low-mass stars or brown dwarfs, as it requires a higher temperature and density than the $[\mathrm{N} \mathrm{II]} \lambda$ $6583 \AA$ line. HH 1158 has strong $\mathrm{H}_{\mathrm{I}}$ lines including the Balmer series $(\mathrm{H} \eta, \mathrm{H} \zeta, \mathrm{H} \delta, \mathrm{H} \gamma, \mathrm{H} \beta, \mathrm{H} \alpha)$, and the Paschen series ( $\mathrm{Pa} 9$, $10,11)$. Also notable is emission in the $\mathrm{Ca}$ II $\mathrm{H}$ and $\mathrm{K}$ lines and the Ca II infrared triplet, typically associated with strong accretion. Another unique feature observed is strong emission in the $\mathrm{O}_{\text {I }} \lambda \lambda 7773,8446 \AA$ atomic lines, which are suggested to be kinematically associated with a strong outflow, and possibly have an origin in high-velocity winds (e.g., Hillenbrand et al. 2012).

The PV diagrams of the continuum-subtracted [O I] $\lambda 6300$, [N II] $\lambda 6584,[\mathrm{~S}$ II] $\lambda 6731$, and $\mathrm{H} \alpha$ lines from the $\mathrm{HH} 1158$ jet in the $90^{\circ}$ spectrum are shown in Figure 3(a). Similar results are obtained in the [O I] $\lambda 6363,\left[\mathrm{~N}_{\mathrm{II}}\right] \lambda 6546,[\mathrm{~S}$ II] $\lambda 6716$ lines. The velocities shown are systemic; we have considered $-30.9 \mathrm{~km} \mathrm{~s}^{-1}$ for the systemic velocity (Riaz et al. 2015). Also shown in Figure 3(a) alongside the PV diagrams are the respective line profiles that have been extracted at a position of $0^{\prime \prime}$ (blue) and -1 !.5 (red). The jet for HH 1158 is spatially resolved at a P. A. of $90^{\circ}$; we see spatially extended emission at this P. A., while the emission is not spatially resolved in the $0^{\circ}$ spectrum. To map the offset of the emission at $0^{\circ}$, we have applied spectro-astrometry and measured offsets of 200 mas in the redshifted jet, and $\sim 50$ mas in the blueshifted jet. This is consistent with the spectrum at $90^{\circ}$ that shows the redshifted emission to be positioned much further from the star than the blueshifted emission. Using the results from the $90^{\circ}$ and $0^{\circ}$ spectra, we constrain the PA of the $\mathrm{HH} 1158$ jet at $102^{\circ} \pm 5^{\circ}$.

HH 1158 exhibits a bipolar asymmetric jet. The blueshifted lobe is brighter and has a slightly higher velocity than the redshifted component. The difference in brightness is well illustrated by the contour plots. Note that the line profiles have been normalized to the peak value since the blue emission is much brighter than the red one, and so the profiles provide information on the relative shape and velocity but not the relative brightness of the components. The line flux ratios in the FELs measured from the $90^{\circ}$ spectrum in the blue and red lobes are approximately [O I]_blue/[O I $]$ red $=67,[\mathrm{~N}$ II $]$ _blue $/[\mathrm{N}$ II $]$ _red $=4$, and $\left[\mathrm{S}_{\mathrm{II}}\right]$ _blue $/\left[\mathrm{S}_{\mathrm{II}}\right]_{\_}$red $=8.5$, which clearly indicate the relative brightness of the blueshifted emission. Using the $\left[\mathrm{S}\right.$ II] line ratios, we derive electron densities, $n_{\mathrm{e}}$, of $4000 \mathrm{~cm}^{-3}$ and $1000 \mathrm{~cm}^{-3}$ in the blue and red lobe, respectively. Therefore, the jet is also asymmetric in density.

The redshifted emission is more spatially extended $\left(\sim 2^{\prime \prime}-3^{\prime \prime}\right)$, compared to the blue component. The jet is much broader in velocity in $\left[\mathrm{O}_{\mathrm{I}}\right]$ than in the $\left[\mathrm{S}_{\mathrm{II}}\right]$ line. The $\left[\mathrm{O}_{\mathrm{I}}\right] \lambda 6300$ line has a larger critical density and traces the jet closer to the source than the other FELs. The [S II] emission, in comparison, can trace the jet knots farther out from the driving source and is more extended. The [O I]_blue line probably has a contribution from a low velocity wind close to the source, which is not seen in the red due to partial obscuration by the disk. This could explain why the blue component is broader than the red lobe. This is also perhaps why we do not see the first knot of redshifted emission until around $1^{\prime \prime}$ from the driving source.

The $\mathrm{H} \alpha$ line shows a broad profile mainly arising from the stellar centroid at $0^{\prime \prime}$ position. The redshifted jet emission seen in the FELs is also detected in the $\mathrm{H} \alpha$ line at $\sim 20-30 \mathrm{~km} \mathrm{~s}^{-1}$, at a position of -1 ". 5 . The $\mathrm{H} \alpha$ line is a better probe of diffuse jet regions and the irradiated portions, therefore some contribution 

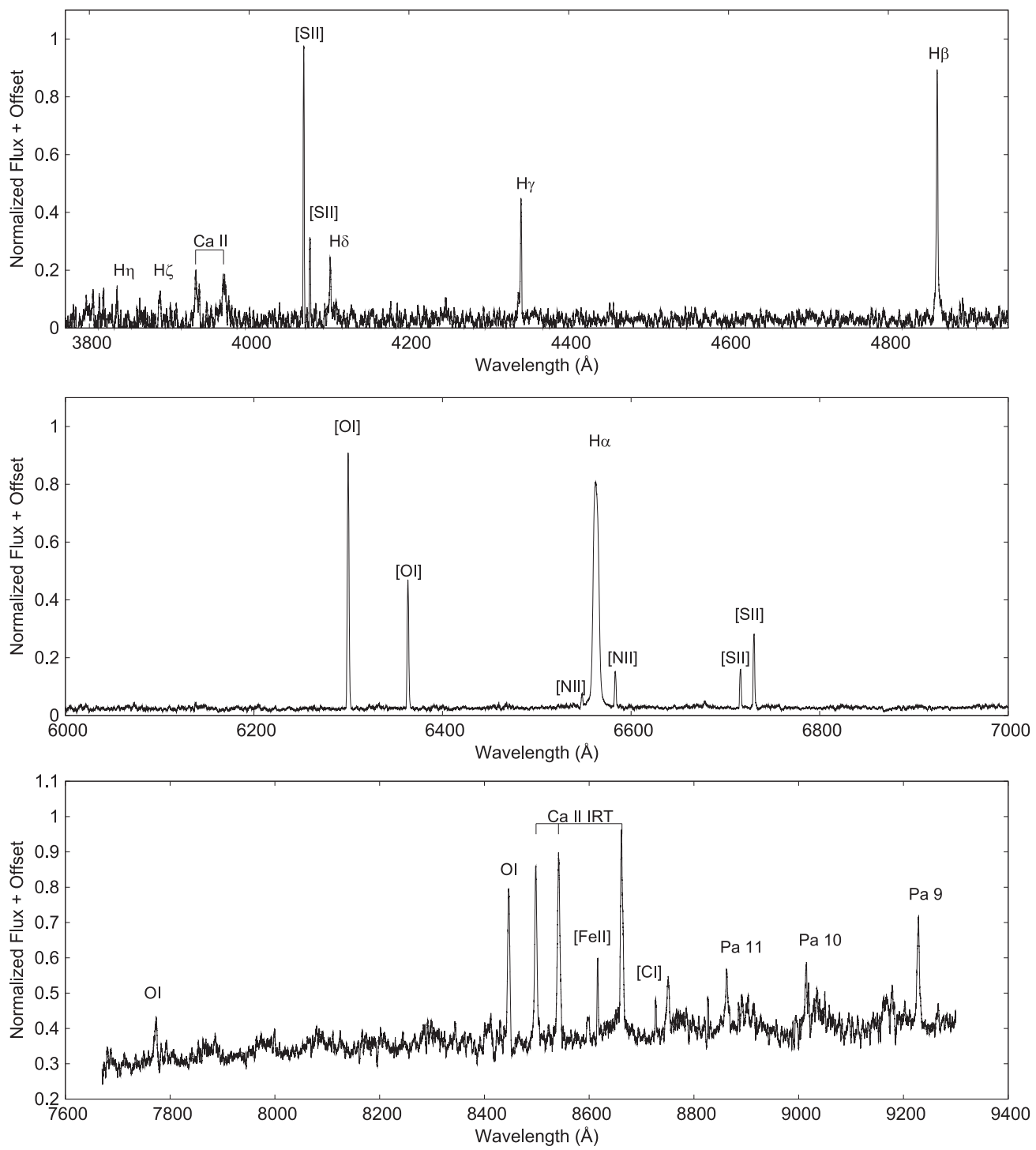

Figure 2. UVES spectra for HH 1158 with the prominent accretion- and outflow-associated emission lines marked.

from the jet emission can be expected. We have conducted a spectro-astrometric analysis in order to investigate the presence of blueshifted jet emission in the wing of the $\mathrm{H} \alpha$ line. Figure 3(b) shows a small offset at the position of the redshifted $\mathrm{H} \alpha$ knot. However, no blueshifted jet emission is seen, and the bulk of the $\mathrm{H} \alpha$ emission is tracing accretion. This is also notable from the broad $\mathrm{H} \alpha$ profile, with a width at $10 \%$ of the line peak of $610 \pm 30 \mathrm{~km} \mathrm{~s}^{-1}$, indicating intense accretion.

We have estimated the mass accretion rate, $\dot{M}_{\text {acc }}$, and the mass outflow rate, $\dot{M}_{\text {out }}$, for $\mathrm{HH} 1158$, by applying the same methods as used in Riaz et al. (2015). We refer to that work for further details on $\dot{M}_{\text {acc }}$ and $\dot{M}_{\text {out }}$ calculation and error estimation. Figure 4 shows $\dot{M}_{\text {acc }}$ derived using multiple accretion activity indicators. The value for $\dot{M}_{\text {acc }}$ derived using the $\left[\mathrm{O}_{\mathrm{I}}\right] \lambda 6300$ line luminosity is about an order of magnitude higher than the mean value. As argued in Riaz et al. (2015), considering that the [O I] line forms above the envelope/disk system, this indirect tracer of accretion is less obscured and could provide a more reliable measure of $\dot{M}_{\text {acc }}$ compared to other diagnostics. The mean $\dot{M}_{\text {acc }}$ for $\mathrm{HH} 1158$, excluding the estimate from the $\left[\mathrm{O}_{\mathrm{I}}\right] \lambda 6300$ line, is measured to be $(3.0 \pm 1.0) \times 10^{-10} M_{\odot} \mathrm{yr}^{-1}$. The mass outflow rate for $\mathrm{HH}$
1158 derived from the [S II] FELs is (5.2 \pm 2.6$) \times 10^{-10} M_{\odot} \mathrm{yr}^{-1}$, respectively. Considering that the $\mathrm{HH} 1158$ jet is also asymmetric in electron density, we estimate $\dot{M}_{\text {out }}$ of $(4 \pm 2) \times 10^{-10} M_{\odot} \mathrm{yr}^{-1}$ in the blue component, and $(1.5 \pm 0.7) \times 10^{-10} M_{\odot} \mathrm{yr}^{-1}$ in the red component, indicating an asymmetry in the outflow rate. The $\dot{M}_{\text {acc }}$ and $\dot{M}_{\text {out }}$ estimates presented in this work are more reliable than the estimates from Riaz et al. (2015). We have used additional accretion indicators, and have obtained spectra along the jet, which provides a better measure of $n_{\mathrm{e}}$, the [S II] fluxes, and the velocities. The high quality of the UVES spectra and a better subtraction of the nebular component also contribute to more accurate estimates. In comparison, the mass loss rates derived for $\mathrm{HH} 444,445$, and 446 are in the range of (2-8) $\times 10^{-7} M_{\odot} \mathrm{yr}^{-1}$ (Andrews et al. 2004). Overall, the accretion and outflow rates for $\mathrm{HH} 1158$ are within the range of $10^{-7}-10^{-10} M_{\odot} \mathrm{yr}^{-1}$ observed among low-mass Class I protostars (e.g., Antoniucci et al. 2011; Hillenbrand et al. 2012), and are comparatively higher than the typical rates of the order of $10^{-10}-10^{-12} M_{\odot} \mathrm{yr}^{-1}$ observed for Class II very low-mass stars and brown dwarfs (e.g., Whelan et al. 2009; Alcalá et al. 2014). 
Table 1

Emission Line Fluxes and Equivalent Widths

\begin{tabular}{|c|c|c|c|}
\hline Line & $\begin{array}{l}\lambda_{\text {central }} \\
(\AA)\end{array}$ & $\begin{array}{c}\text { Line Flux } \\
\left(10^{-15} \mathrm{erg} \mathrm{cm}^{-2} \mathrm{~s}^{-1}\right)\end{array}$ & $\begin{array}{c}\text { Equivalent Width } \\
(\AA)\end{array}$ \\
\hline $\mathrm{H} \eta$ & 3835.38 & $1.93 \pm 0.5$ & $-34.9 \pm 2.0$ \\
\hline $\mathrm{H} \zeta$ & 3889.05 & $1.74 \pm 0.5$ & $-45 \pm 10$ \\
\hline Ca II $\mathrm{K}$ & 3933.66 & $2.5 \pm 0.3$ & $-20.4 \pm 4.0$ \\
\hline $\mathrm{H} \epsilon+\mathrm{Ca}$ II $\mathrm{H}$ & 3970.07 & $1.9 \pm 0.3$ & $-7.4 \pm 0.3$ \\
\hline [S II] & 4068.6 & $8.2 \pm 1.0$ & $-45.8 \pm 5.0$ \\
\hline [S II] & 4076.3 & $2.5 \pm 0.5$ & $-30.0 \pm 10.0$ \\
\hline $\mathrm{H} \delta$ & 4101.74 & $1.5 \pm 2.5$ & $-2.5 \pm 1.5$ \\
\hline $\mathrm{H} \gamma$ & 4340.47 & $3.4 \pm 0.5$ & $-15.7 \pm 8$ \\
\hline $\mathrm{H} \beta$ & 4861.33 & $10.6 \pm 3$ & $-23.6 \pm 10$ \\
\hline$[\mathrm{O}$ I] & 6300.30 & $36.8 \pm 3.0$ & $-250 \pm 130$ \\
\hline [O I $]$ & 6363.78 & $16.0 \pm 2.0$ & $-25.6 \pm 5.0$ \\
\hline [N II $]$ & 6548.0 & $1.5 \pm 0.1$ & $-3.2 \pm 0.1$ \\
\hline $\mathrm{H} \alpha$ & 6562.85 & $98.2 \pm 5.0$ & $-102.5 \pm 20.0$ \\
\hline [N II $]$ & 6583.45 & $4.0 \pm 0.6$ & $-9.7 \pm 3.0$ \\
\hline$[\mathrm{S}$ II $]$ & 6716.44 & $4.4 \pm 0.3$ & $-17.1 \pm 3.5$ \\
\hline [S II] & 6730.82 & $7.6 \pm 0.5$ & $-15.1 \pm 1.0$ \\
\hline $\mathrm{O}_{\mathrm{I}}$ & 7773.0 & $1.06 \pm 0.2$ & $-2.6 \pm 0.3$ \\
\hline $\mathrm{O}_{\mathrm{I}}$ & 8446.36 & $3.3 \pm 0.5$ & $-7.4 \pm 0.5$ \\
\hline $\mathrm{Ca}$ II & 8498.02 & $3.5 \pm 1.0$ & $-7.2 \pm 2.0$ \\
\hline Ca II & 8542.09 & $5.1 \pm 1.0$ & $-12.7 \pm 2.0$ \\
\hline [Fe II] & 8616.95 & $0.8 \pm 0.1$ & $-1.7 \pm 0.2$ \\
\hline $\mathrm{Ca}$ II & 8662.14 & $4.7 \pm 1.0$ & $-10.4 \pm 3.0$ \\
\hline$\left[\mathrm{C}_{\mathrm{I}}\right]$ & 8727.1 & $0.4 \pm 0.3$ & $-0.7 \pm 0.3$ \\
\hline H I (Pa 11) & 8863.4 & $1.2 \pm 0.2$ & $-2.12 \pm 0.6$ \\
\hline $\mathrm{H}_{\mathrm{I}}(\mathrm{Pa} 10)$ & 9015.6 & $0.66 \pm 0.1$ & $-1.12 \pm 0.2$ \\
\hline $\begin{array}{l}\mathrm{H} \mathrm{I}_{\text {I }}(\mathrm{Pa} \mathrm{9)} \\
\quad+[\mathrm{Fe} \text { II }]\end{array}$ & 9229.7 & $2.22 \pm 0.4$ & $-4.02 \pm 0.4$ \\
\hline
\end{tabular}

\section{DISCUSSION: SIMILARITIES WITH HH 444-HH 447 JETS IN $\sigma$ ORIONIS}

A detailed study of the irradiated HH 444-HH 447 outflows and their driving sources was presented in Andrews et al. (2004), using high-resolution spectroscopy from the Keck HIRES spectrograph. All of these previously known $\sigma$ Ori jets demonstrate a jet/counterjet brightness asymmetry. In particular, the PV diagrams for $\mathrm{HH} 444,445$, and 446 show a bipolar asymmetric jet morphology, where the peak in emission is blueshifted for all FELs. The blueshifted lobe is brighter, denser, and is at a higher velocity than the redshifted component. There is either a very weak redshifted lobe or an extended redshifted wing, and in all cases it is significantly fainter than the blueshifted lobe. Several discrete knots of roughly the same velocity and intensity are also seen in the blueshifted branches in the FEL emission for these jets. Faint, extended emission is also observed in the $\mathrm{H} \alpha$ line for $\mathrm{HH} 445$ and 446. HH 447 is the weakest among these four jets and exhibits a similar jet/counterjet structure; however, only a redshifted branch is observed for this jet with no evidence of a blueshifted component.

The PA for the blue lobes in these jets are $70^{\circ}$ for $\mathrm{HH} 444$, $100^{\circ}$ for $\mathrm{HH} 445,166^{\circ}$ for $\mathrm{HH} 446$, and $210^{\circ}$ for $\mathrm{HH} 447$ (Andrews et al. 2004). As can be visualized from Figure 1, the weaker outflow component for all of these $\mathrm{HH}$ jets is the beam oriented toward the west and is exposed to the ionizing radiation from $\sigma$ Ori, resulting in a higher extent of photoevaporation, whereas the eastward beam facing away from $\sigma$ Ori is much brighter than the counter jet. For the case of $\mathrm{HH}$ 1158 , the jet PA is estimated to be $102^{\circ}$, which would imply that the brighter, blueshifted component is oriented toward the south-east of the central source, while the weaker red component is oriented toward the north-west. HH 1158 appears to be a case more similar to HH 446 and HH 447. The orientation of these jets is such that both lobes are exposed toward $\sigma$ Ori, and thus probably receive equal amounts of radiation, unlike the more extreme cases of $\mathrm{HH} 444$ and 445. Yet there are clear asymmetries seen for these jets, notably for HH 447 where the blue lobe is photoevaporated to such an extent that it was never detected. It may be the case that the brighter component is somewhat protected by the circumstellar disk from the external radiation, whereas the weaker component, which also appears to be more tilted toward $\sigma$ Ori for these three jets (Figure 1), has been exposed to a higher dose of photoionization from the massive stars. That the brighter jet component is probably protected to some extent by the disk has also been suggested for HH 446 (Andrews et al. 2004).

All of the features seen in the HH 444-447 and HH 1158 jets are also observed in non-irradiated jets. A distinction between asymmetric irradiated and non-irradiated micro-jets is more difficult to make for $\mathrm{HH}$ objects in $\mathrm{H}$ II regions, where it is easy to suspect a bright rim or compact globule to be an HH bow shock. In such cases, a criteria used for irradiated jets is to have a $\mathrm{H} \alpha /\left[\mathrm{S}_{\mathrm{II}}\right]$ ratio that is clearly larger than one, indicating enhanced intensity of the $\left[\mathrm{S} \mathrm{II}_{\mathrm{II}}\right.$ and [N II] FELs with respect to $\mathrm{H} \alpha$ emission (e.g., Reipurth \& Bally 2003; Reipurth et al. 2010; Comerón et al. 2013). Considering the redshifted knot in the $\mathrm{H} \alpha$ emission for $\mathrm{HH} 1158$, the $\mathrm{H} \alpha /\left[\mathrm{S}_{\text {II }}\right]$ ratio is 2.8 . The value for this ratio is many times $>1$ for HH 444-447 jets (Reipurth et al. 1998; Reipurth \& Bally 2001, 2003). While this is not an affirmative test for irradiated jets, the $\mathrm{H} \alpha /\left[\mathrm{S}_{\mathrm{II}}\right]$ ratio being significantly larger than the background value indicates that the blobs of material observed in an irradiated region are related to shocked material. The irradiated environment in the $\sigma$ Ori region being dominated by the UV radiation field of the massive OB stars is a strong argument to suggest that environmental effects are the main cause of the asymmetries observed for the HH 444-HH 447 and HH 1158 jets.

$\mathrm{HH} 1158$ fits into the overall picture observed for the $\mathrm{HH}$ 444-HH 447 jets, with the evidence being that (a) it shows bipolar asymmetric jet morphology, with the lobe tilted toward $\sigma$ Ori being the weaker component. The asymmetries are also observed in the brightness, density, and velocity of the lobes, similar to HH 444-447; (b) it lies in close proximity to $\sigma$ Ori, closer than $\mathrm{HH} 447$, to be "bathed" in the UV radiation field and termed as an irradiated jet. The high value for the $\mathrm{H} \alpha /\left[\mathrm{S}_{\text {II }}\right]$ ratio is also consistent with the ratios measured in other irradiated jets, including HH 444-447; (c) the driving source for HH 1158 is not an embedded but an optically visible source, with the jet emission seen brightly in optical lines. The driving sources for HH 444-HH 447 are suggested to be of mid-K to early-M spectral types (Andrews et al. 2004), corresponding to bolometric luminosities higher than $\sim 0.5 L_{\odot}$. This makes $\mathrm{HH}$ 1158 the lowest luminosity analog of externally irradiated $\mathrm{HH}$ jets identified yet in the $\sigma$ Orionis cluster.

A search through the literature shows that this is the lowest mass case among all known irradiated $\mathrm{HH}$ jets. The presence of an extended collimated jet that is bipolar and the evidence of shocked emission knots make HH 1158 a unique new HH jet at the very low-luminosity end. The comparison with $\mathrm{HH}$ 444-447 presented here suggests that HH 1158 is a scaled down case of irradiated jets driven by YSOs. Therefore, 

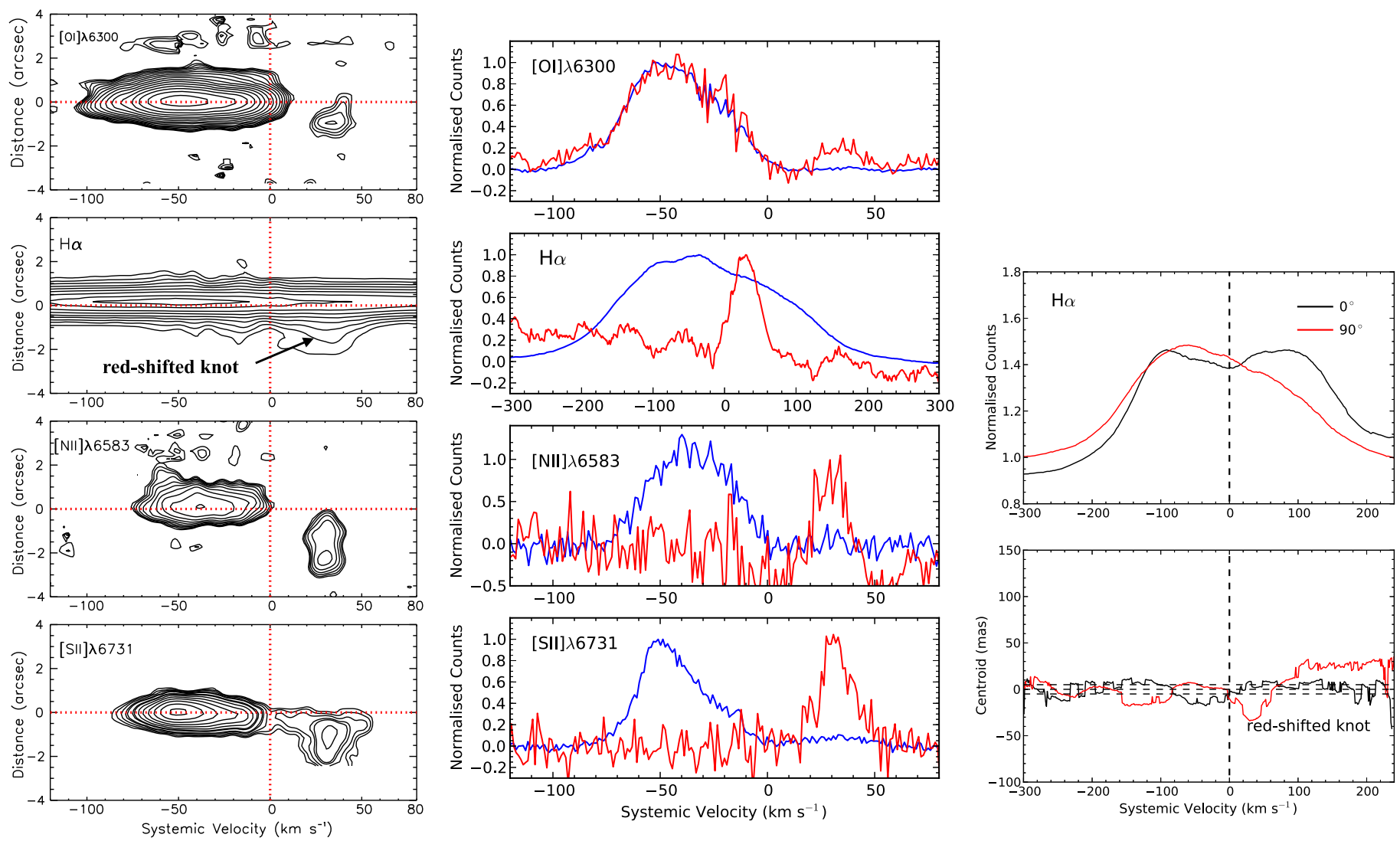

Figure 3. Left (a): the PV plots for HH 1158 alongside the line profiles, constructed from the $90^{\circ}$ spectra. The line profiles are extracted from a position of $0^{\prime \prime}$ and $-1 "$. 5 . The extracted lines have been normalized to the line peaks. Right (b): spectro-astrometric analysis of the HH $1158 \mathrm{H} \alpha$ line. The spectra have been binned to increase the $\mathrm{S} / \mathrm{N}$ and the spectro-astrometric accuracy. The horizontal lines delinates the $\pm 1 \sigma$ uncertainty. A small offset is seen at $\sim 20 \mathrm{~km} \mathrm{~s}{ }^{-1}$, which is the redshifted lobe of the jet.

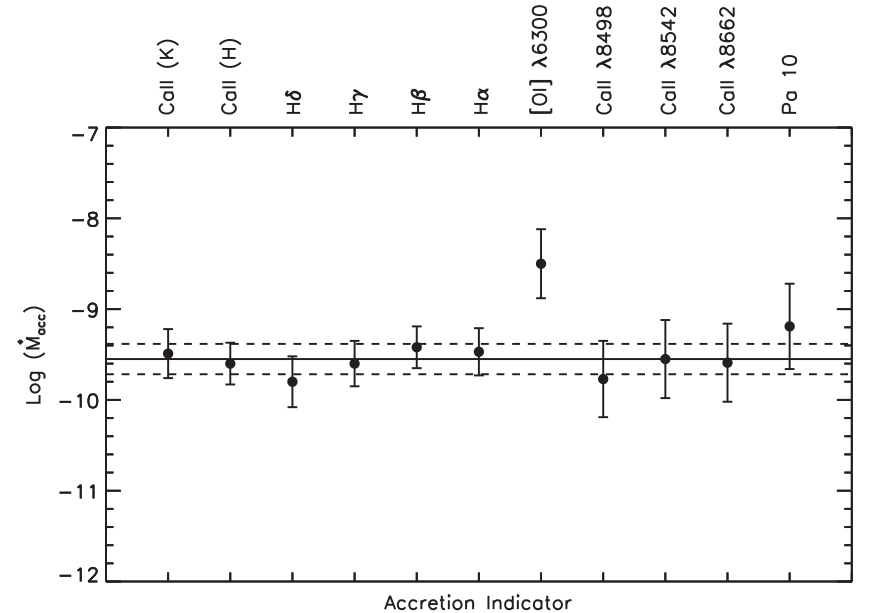

Figure 4. Accretion rates $\log \dot{M}_{\text {acc }}$ in $M_{\odot} \mathrm{yr}^{-1}$ derived from various line diagnostics (labeled) for $\mathrm{HH} 1158$. Horizontal line indicates the mean level calculated without including the [O I] line.

irradiated jets can now be added to the list of properties which jets driven by the lowest luminosity objects have in common with protostellar jets.

\section{SUMMARY}

We have identified a new externally irradiated jet, HH 1158 , in $\sigma$ Orionis, the overall properties of which are similar to the previously known irradiated $\mathrm{HH}$ jets in this cluster. $\mathrm{HH} 1158$ is driven by an optically visible Class Flat source, and shows bipolar asymmetric jet morphology, with the weaker jet beam tilted toward the massive stars in the cluster. There are also asymmetries observed in the brightness, density, and velocity of the lobes. Due to the close proximity to $\sigma$ Ori, the asymmetry is likely caused by the irradiated environment in the cluster. HH 1158 is the lowest luminosity analog of externally irradiated $\mathrm{HH}$ jets identified yet in the $\sigma$ Orionis cluster, and is the lowest luminosity case among all known irradiated $\mathrm{HH}$ jets.

We thank B. Reipurth, J. Bally, and J. Caballero for their suggestions on the nature of this object. B.R. acknowledges funding from the Marie Sklodowska-Curie Individual Fellowship (Grant Agreement No. 659383). E. T. Whelan acknowledges financial support from the Deutsche Forschungsgemeinschaft through the Research Grant Wh 172/1-1. Based on observations collected with UVES at the Very Large Telescope on Cerro Paranal (Chile), operated by the European Southern Observatory.

\section{REFERENCES}

Alcalá, J. M., Natta, A., Manara, C. F., et al. 2014, A\&A, 561, A2 Andrews, S. M., Reipurth, B., Bally, J., \& Heathcote, S. R. 2004, ApJ, 606,353

Antoniucci, S., García López, R., Nisini, B., et al. 2011, A\&A, 534, 32

Comerón, F., Pasquali, A., \& Alves de Oliveira, C. 2013, A\&A, 552, A30 
Greene, T. P., Wilking, B. A., Andre, P., Young, E. T., \& Lada, C. J. 1994, ApJ, 434, 614

Hillenbrand, L. A., Knapp, G. R., Padgett, D. L., Rebull, L. M., \& McGehee, P. M. 2012, AJ, 143, 37

Reipurth, B., \& Bally, J. 2001, ARA\&A, 39, 403

Reipurth, B., \& Bally, J. 2003, AJ, 126, 893

Reipurth, B., Bally, J., Fesen, R. A., \& Devine, D. 1998, Natur, 396, 343

Reipurth, B., Aspin, C., Bally, J., Tobin, J. J., \& Walawender, J. 2010, AJ, 140,699
Riaz, B., Thompson, M., Whelan, E. T., \& Lodieu, N. 2015, MNRAS, 446, 2550

Walter, F. M., Sherry, W. H., Wolk, S. J., \& Adams, N. R., Handbook of Star Forming Regions, Vol. I: The Northern Sky, ed. B. Reipurth (ASP Monograph Publ., Vol. 4; San Francisco, CA: ASP), 732

Whelan, E. T., \& Garcia, P. 2008, LNP, 742, 123

Whelan, E. T., Ray, T. P., Podio, L., Bacciotti, F., \& Randich, S. 2009, ApJ, 706, 1054

Whelan, E. T., Ray, T. P., Bacciotti, F., et al. 2005, Natur, 435, 652 